\title{
Pseudogaps in Nested Antiferromagnets
}

\author{
C. Pépin ${ }^{1}$ and M. R. Norman ${ }^{2}$ \\ ${ }^{1}$ SPhT, L'Orme des Merisiers, CEA-Saclay, 91191 Gif-sur-Yvette, France \\ ${ }^{2}$ Materials Science Division, Argonne National Laboratory, Argonne, Illinois 60439
}

(Dated: November 13, 2018)

\begin{abstract}
We analyze the fluctuation corrections to magnetic ordering in the case of a 3D antiferromagnet with flat Fermi surfaces, as physically realized in the case of chromium, and find that they are insufficient to produce a quantum critical point. This implies that the critical point observed in vandium doped chromium is due to a loss of nesting. We also derive the fermion self-energy in the paramagnetic phase and find that a pseudogap exists, though its magnitude is significantly reduced as compared to the spectral gap in the ordered state in the limit where the latter is small in comparison to the Fermi energy.
\end{abstract}

PACS numbers: $75.30 . \mathrm{Fv}, 71.10 . \mathrm{Hf}, 78.20 . \mathrm{Bh}$

The subject of magnetic quantum critical points has sparked much interest in the physics community. In the case of heavy fermion metals, this interest has largely been motivated by the observation that Fermi liquid theory breaks down in the vicinity of such critical points 1 . At present, the theory behind this breakdown is not well understood because of the strong coupling nature of the Kondo lattice problem ${ }^{2}$.

Recently, Yeh et $a l^{3}$. have studied the more straightforward case of vanadium doped chromium. Chromium is the classic example of a spin density wave magnet driven by Fermi surface nesting ${ }^{4}$. Upon doping with vanadium, the Néel temperature, $T_{N}$, is rapidly suppressed to zero. Motivated by the speculation that the Hall number may jump at a magnetic quantum critical point due to Fermi surface rearrangement ${ }^{2}$, the authors of Ref. 3 studied the Hall conductivity and indeed identified such a jump. Moreover, they found a strong temperature dependence of the Hall number, which they speculated was due to the presence of a pseudogap near the critical point. The corresponding signature of this pseudogap has been looked for as a spin gap in the dynamic susceptibility, but so far results are inconclusive $e^{\frac{5}{2}}$.

The simplicity of the case of vanadium doped chromium obviously begs for a theoretical treatment. Recently, it has been shown that the jump of the Hall number can be understood as due to the sudden removal of flat parts of the Fermi surface upon magnetic ordering 6 . The presence of flat Fermi surfaces obviously points to the possibility of a pseudogap, given the quasi-1D nature of the fermion dispersion? ${ }^{7}$.

In this paper, we consider the flat Fermi surface model of chromium originally proposed by Shibatani et al 을 where the Fermi surface is approximated as a cube. We find, in agreement with earlier work ${ }^{9}$, that fluctuation corrections are less singular than in a non nested antiferromagnet, and thus are insufficent to drive the Néel temperature to zero. Rather, the critical temperature must be driven to zero by loss of Fermi surface nesting 10 . This is consistent with recent pressure data 11 , which indicate scaling exponents near the critical point for the Néel temperature and Hall number in agreement with analytic results based on a curved Fermi surface $e^{6}$. Moreover, we evaluate the fermion self-energy in the paramagnetic phase, and indeed find a pseudogap in the spectral function. This pseudogap, though, scales as $T_{N}^{3} / E_{F}^{2}$ for $T_{N} \ll E_{F}$, where $E_{F}$ is the Fermi energy, and thus is likely to be too small to be responsible for the strong temperature variation of the Hall number ${ }^{3}$. Instead, the observed variation may be due to the temperature dependence of the inelastic scattering rate, as observed in other transition metals 12 . On the other hand, for more strongly correlated systems where $T_{N} \sim E_{F}$, then the pseudogap does scale as $T_{N}$.

The polarization bubble for the flat case is identical to that of the BCS theory for superconductivity $\stackrel{13}{ }$. That is,

$$
\chi_{0}=N\left[\ln (1.14 D / T)-\xi^{2} q^{2}+i \alpha \omega\right]
$$

where $D$ is the ultraviolet cut-off (bandwidth) and $N$ the density of states. The expansion coefficient $\xi$ is isotropic for the commensurate case $(Q=2 \pi / a$ for chromium), and weakly anisotropic for the incommensurate case ${ }^{13}(q$ is defined relative to the ordering wavevector, $Q$ ). In linear response theory, the interacting susceptibility is

$$
\chi_{M F}=\chi_{0} /\left(1-g \chi_{0}\right)
$$

where $g$ is the exchange interaction. The zero of this denominator defines the mean field transition temperature, $T_{M F}=1.14 D e^{-1 / g N}$. The mean field inverse susceptibility is then

$$
\chi_{M F}^{-1}=\frac{N g}{\chi_{0}}\left[\ln \frac{T}{T_{M F}}+\xi^{2} q^{2}-i \alpha \omega\right]
$$

Fluctuation corrections in the Hartree approximation give a true inverse susceptibility of 14

$$
\chi^{-1}=\chi_{M F}^{-1}+b<M^{2}>,
$$

with $b$ diverging as $1 / T^{2}, \alpha$ as $1 / T$, and $\xi$ as $1 / T$. The system undergoes a transition towards antiferromagnetic order at a temperature $T_{N}$ for which $\chi^{-1}(\omega=0, q=$ $0)=0$. The important difference of the nesting case to 
a normal 3D antiferromagnet is the temperature dependence of the expansion coefficients. Because of this, spin fluctuations become relatively less important as the temperature is lowered, and thus fluctuation effects are less singular ${ }^{9}$.

To see this, we approximate $\left\langle M^{2}>\right.$, the fluctuating staggered moment, by its classical value $(\omega \ll T)$

$$
<M^{2}>=\frac{T}{2} \int \frac{d^{3} q}{(2 \pi)^{3}} \chi(q, 0)=\frac{T q_{c} \chi_{0}}{4 \pi^{2} N g \xi^{2}}
$$

The latter equality assumes that $T=T_{N}$. If $q_{c}$, the classical cut-off, is assumed to satisfy the condition $\Gamma\left(q_{c}\right)=$ $T$, then one can show that the classical value is approximately equal to the true quantum mechanical value for $<M^{2}>14$. Here, $\Gamma$ is the frequency half width of the dynamic susceptibility, which, at $T=T_{N}$ is $q_{c}=\sqrt{\alpha T} / \xi$. Using this, $<M^{2}>$ reduces to

$$
<M^{2}>=\frac{T^{4} \sqrt{\alpha^{\prime}} \chi_{0}}{4 \pi^{2} N g \xi^{\prime 3}}
$$

where $\alpha^{\prime}=\alpha T$ and $\xi^{\prime}=\xi T$ are temperature independent constants. Recognizing that $b^{\prime}=b T^{2}$ is also a temperature independent constant, $\chi^{-1}(0,0)$ reduces to

$$
\chi^{-1}(0,0)=\ln \frac{T}{T_{M F}}+\frac{b^{\prime} \sqrt{\alpha^{\prime}} T^{2} \chi_{0}^{2}}{4 \pi^{2} N^{2} g^{2} \xi^{\prime 3}}
$$

That is, the correction to $T_{M F}$ goes as $T^{2} \ln ^{2} T$, which is less singular than the $T^{3 / 2}$ correction for an ordinary $3 \mathrm{D}$ antiferromagnet 15 . Our result agrees with earlier results of Hasegawa ${ }^{9}$, though our derivation is more straightforward.

Since $T_{N}$ can never be driven to zero for a perfectly flat Fermi surface (due to the logarithmic divergence of $\chi_{0}$ ), then one might think that the quantum critical point is probably not controlled by fluctuations 16 . Rather, loss of nesting is the likely cause of the quantum critical point. This is consistent with band theory results ${ }^{6}$, which find an increasing mismatch of the electron and hole octahedral surfaces as the hole doping is increased. Such warping corrections will cause the fluctuation corrections to cross over to the standard 3D antiferromagnetic result near the critical point.

Having addressed the question of fluctuations, we now turn to the question of the pseudogap. Postulating a pseudogap in the case of nesting is quite natural given the quasi-1D nature of the fermion dispersion. On the other hand, as we have seen above, despite this quasi-1D behavior, the spin fluctuation spectrum is still 3D-like, and this raises questions about how strong the pseudogap effect will be. To address this, we derive the fermionic self-energy to lowest order. We note that this is given by a convolution of an effective interaction $V$ with the fermion Greens function, where ${ }^{17}$

$$
V=g+g^{2} \chi+g^{2} \chi /\left(1+g \chi_{0}\right) \simeq(3 g / 2) \chi / \chi_{0}
$$

with the first term the bare interaction, the second one from summing a ladder series, and the third from summing a bubble series. The most singular part of the fermionic self-energy comes from the classical fluctuations, and can be approximated as 18

$$
\Sigma=\frac{3 T}{2 N} \int \frac{d^{3} q}{(2 \pi)^{3}} \frac{1}{A+\xi^{2} q^{2}} \frac{1}{\omega-\epsilon_{k+Q+q}}
$$

where $\epsilon$ is the fermionic dispersion and $A=\ln \frac{T}{T_{M F}}+$ $\frac{b \chi_{0}}{N g}<M^{2}>$. In the flat case, $\epsilon_{k+Q+q}=-\epsilon_{k}-v q_{\|}$, where $v$ is the Fermi velocity and $q_{\|}$is normal to the flat surface. $\Sigma$ now becomes

$$
\Sigma=\frac{3 T}{2 N} \int \frac{d q_{\|} d^{2} q_{\perp}}{(2 \pi)^{3}} \frac{1}{A+\xi^{2}\left(q_{\|}^{2}+q_{\perp}^{2}\right)} \frac{1}{\omega+\epsilon_{k}+v q_{\|}}
$$

The integral over $q_{\perp}$ gives $\left(\xi^{2} q_{c}^{2}=\alpha T\right)$

$$
\Sigma=\frac{3 T}{8 \pi N \xi^{2}} \int \frac{d q_{\|}}{2 \pi} \ln \left(\frac{A+\xi^{2} q_{\|}^{2}+\alpha T}{A+\xi^{2} q_{\|}^{2}}\right) \frac{1}{\omega+\epsilon_{k}+v q_{\|}}
$$

The $q_{\|}$integral is convergent, and the cut-off can be taken to infinity. The result is

$$
R e \Sigma=\frac{3 T}{8 \pi N v \xi^{2}}\left(\tan ^{-1} \frac{\xi\left(\omega+\epsilon_{k}\right)}{v \sqrt{A}}-\tan ^{-1} \frac{\xi\left(\omega+\epsilon_{k}\right)}{v \sqrt{A+\alpha T}}\right)
$$

$$
\operatorname{Im} \Sigma=\frac{3 T}{16 \pi N v \xi^{2}} \ln \frac{A+(\xi / v)^{2}\left(\omega+\epsilon_{k}\right)^{2}+\alpha T}{A+(\xi / v)^{2}\left(\omega+\epsilon_{k}\right)^{2}}
$$

To understand these expressions further, we assume that $T=T_{N}(A=0)$ where the pseudogap effect should be most pronounced. This yields

$$
\begin{gathered}
\operatorname{Re} \Sigma=\frac{\bar{\Delta}^{2}}{2.7 T_{N}}\left(\frac{\pi}{2} \operatorname{sgn}\left(\omega+\epsilon_{k}\right)-\tan ^{-1} \frac{\omega+\epsilon_{k}}{2.7 T_{N}}\right) \\
\operatorname{Im} \Sigma=\frac{\bar{\Delta}^{2}}{5.4 T_{N}} \ln \left(1+\left(\frac{2.7 T_{N}}{\omega+\epsilon_{k}}\right)^{2}\right)
\end{gathered}
$$

where a typical energy scale $\bar{\Delta}$ is defined as

$$
\bar{\Delta}^{2}=8.1 T_{N}^{4} /\left(8 \pi N v \xi^{\prime 2}\right)
$$

These were obtained by noting that for the flat case $\mathrm{e}^{9}$, $\alpha^{\prime}=\pi / 8$ and $\xi^{\prime}=v \sqrt{7 \xi(3) /\left(16 \pi^{2}\right)}$, where $\xi(3)=1.202$. We note that $2.7 T_{N}$ is the natural frequency scale of the problem, and that $\bar{\Delta}$ has units of energy.

The fermion spectral function is given as

$$
\mathcal{A}(k, \omega)=\frac{1}{\pi} \frac{\operatorname{Im} \Sigma}{\left(\omega-\epsilon_{k}-\operatorname{Re} \Sigma\right)^{2}+(\operatorname{Im} \Sigma)^{2}}
$$

In Fig. 1, we show the spectral function at the Fermi surface for two cases, $\bar{\Delta}=2.7 T_{N}$ and $0.27 T_{N}$. In the first 

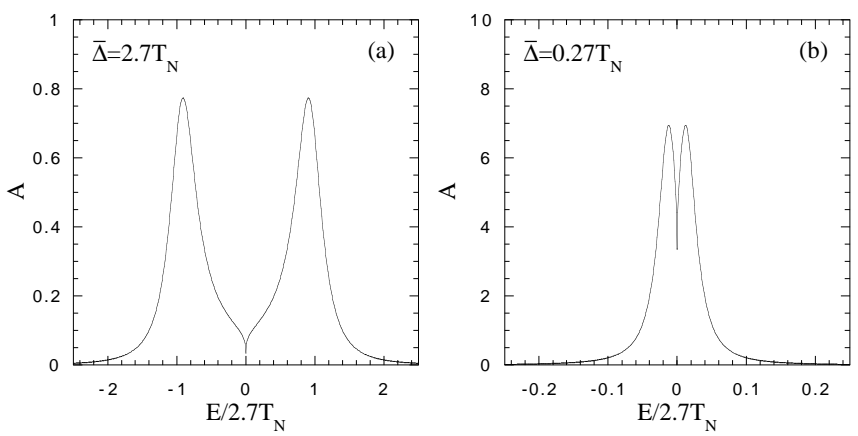

FIG. 1: Spectral function, $\mathcal{A}$, on the Fermi surface derived using Eqs. 14-17 for two values of $\bar{\Delta}$. Note differing energy scales in the two plots.

case (strong coupling limit), the spectral gap is approximately equal to $\bar{\Delta}$. In the second case (more appropriate for chromium as will be seen below), the spectral gap is significantly smaller than $\bar{\Delta}$. In Fig. 2, we plot the spectral gap obtained from half the spectral peak to peak separation in Fig. 1 versus $\bar{\Delta}$. For large $\bar{\Delta}$ (comparable to $T_{N}$ ), the spectral gap scales with $\bar{\Delta}$, whereas for small $\bar{\Delta}$, the spectral gap scales quadratically with $\bar{\Delta}$.

To understand these results analytically, we note that the pole of the fermion Greens function on the Fermi surface is given by the condition $\omega-\operatorname{Re} \Sigma(\omega)=0$. Let us first assume this pole energy is of order $2.7 T_{N}$. Then under these conditions, $\operatorname{Re} \Sigma$ in Eq. 12 can be approximated as (high frequency expansion)

$$
\operatorname{Re} \Sigma_{h i g h}=\frac{\bar{\Delta}^{2}}{\omega}
$$

This expression is identical to the BCS expression for the self-energy, and the pole can easily be seen to occur at an energy $\bar{\Delta}$. That is, there is a spectral gap equal to $\bar{\Delta}$, and this explains the behavior for large $\bar{\Delta}$ in Fig. 2. By noting that the density of states for the flat case, $N$, is $1 /\left(2 \pi v a^{2}\right)$, then

$$
\bar{\Delta}=6.2 T_{N}^{2} a / v
$$

Since $v / a \sim E_{F}$ ( $a$ is the lattice constant), this would imply that $T_{N}$ must be of order the Fermi energy, $E_{F}$, for this high frequency approximation to be valid. This is not satisfied for chromium, since $T_{N} \ll E_{F}$ in that case.

In the other limit, one expands the self-energy for small $\omega$, obtaining (low frequency expansion)

$$
\operatorname{Re} \Sigma_{\text {low }}=\frac{\bar{\Delta}^{2}}{2.7 T_{N}}\left(\frac{\pi}{2} \operatorname{sgn}(\omega)-\frac{\omega}{2.7 T_{N}}\right)
$$

The pole energy is then given approximately by $\frac{\pi}{2} \bar{\Delta}^{2} /\left(2.7 T_{N}\right)$. Therefore, for the case where $T_{N} \ll E_{F}$, then the spectral gap scales as $T_{N}^{3} / E_{F}^{2}$.

Pure chromium exhibits a classic mean field transition as far as specific heat measurements are concerned ${ }^{4}$. This implies that it is in the weak coupling limit, consistent

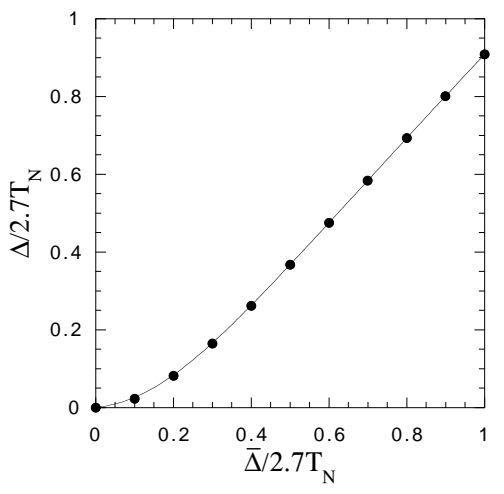

FIG. 2: Spectral gap, $\Delta$, (half the peak to peak separation in Fig. 1) versus $\bar{\Delta}$ derived using Eqs. $14-17$. Note quadratic behavior of the spectral gap for small $\bar{\Delta}$ and linear behavior for large $\bar{\Delta}$.

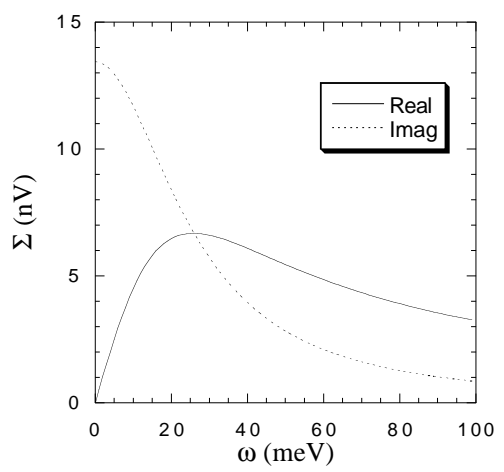

FIG. 3: Self-energy on the Fermi surface calculated from Eqs. 12-13 using neutron scattering parameters from $\mathrm{Cr}-\mathrm{V}$ $5 \%$. Although the $\omega$ scale is reasonable, the magnitude of $\Sigma$ is so small (nanovolts) that a pseudogap does not develop.

with the small ratio of $T_{N}$ to $E_{F}$. We can quantify this by using parameters extracted from neutron scattering data. In Ref. 5, the authors use a form for the susceptibility identical to the one employed here

$$
\chi(q, \omega)=\frac{\chi_{Q}}{1+q^{2} / \kappa_{0}^{2}-i \omega / \omega_{s f}}
$$

By comparing to our expressions, we see that $\kappa_{0}^{2}=A / \xi^{2}$ and $\omega_{s f}=A / \alpha$. Using this, the prefactor outside the parenthesis in Eq. 12 becomes $6 T^{2} \kappa_{0}^{2} a^{2} /\left(\pi \omega_{s f}\right)$ and the quantities dividing $\left(\omega+\epsilon_{k}\right)$ in the $\tan ^{-1}$ functions in Eq. 12 are $2.7 \sqrt{\omega_{s f} T}$ and $2.7 \sqrt{\omega_{s f} T+T^{2}}$ respectively. For $\mathrm{Cr}-\mathrm{V} 5 \%, \kappa_{0}=0.11 \AA^{-1}$ and $\omega_{s f}=88 \mathrm{meV}$ for $\mathrm{T}=12 \mathrm{~K}(a=2.88 \AA)$. We plot the resulting self-energy from Eqs. 12 and 13 in Fig. 3. Though the $\omega$ structure of $\Sigma$ is reasonable (looking like a damped version of Eq. 18 with a maximum in $\operatorname{Re} \Sigma$ at $26 \mathrm{meV}$ ), the value of $\Sigma$ itself (nanovolts) is far too small to cause a pseudogap.

Based on this, we expect only weak pseudogap effects, even in the magnetically ordered part of the phase diagram. We note that our derivation of the self-energy assumes that the spin structure factor is quasi-static, a 


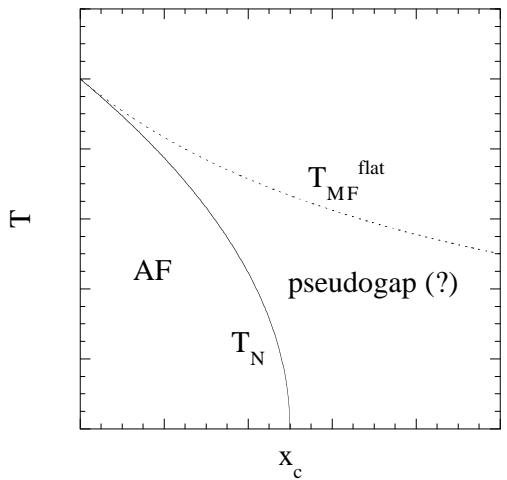

FIG. 4: Illustration of a possible scenario for the pseudogap phase in the $\mathrm{V}$ doped $\mathrm{Cr}$ system. $T_{M F}^{\text {flat }}$ is the mean field temperature for a flat Fermi surface, $T_{N}$ the actual transition temperature which is suppressed by loss of nesting (warping of the Fermi surface). The pseudogap, if it exists, should be confined to the region between these two temperatures.

property of the renormalized classical regime $\underline{\underline{18}}$. That is, we would not necessarily expect pseudogap effects in the quantum critical regime. This conclusion is bolstered by our evalulation of quantum corrections to the self-energy, which we do not find to be singular.

On the other hand, in the flat model, $T_{M F}$ never vanishes as a function of doping. It is a loss of nesting which leads to the quantum critical point. Therefore, it is possible that a pseudogap exists for all dopings which satisfy $T_{N}<T<T_{M F}$. This is illustrated in Fig. 4. Still, we expect that although pseudogap effects are possible near the quantum critical point of vanadium doped chromium, they are likely to be weak. They could perhaps be best searched for by photoemission, which sees the spectral gap quite easily in pure chromium ${ }^{19}$.

This begs the question of what is responsbile for the strong temperature dependence of the Hall number ob- served by Yeh et al $\underline{3}$ which occurs even for dopings far beyond the quantum critical point. The Hall number is temperature dependent in transition metals such as $\mathrm{Cu}$, which can be attributed to the temperature dependence of the electron-phonon scattering rate $\frac{12}{2}$. For the vanadium doped chromium case, this would be consistent with the $T^{3}$ dependence of the resistivity ${ }^{\underline{3}}$, which points to the prevalence of electron-phonon effects. Moreover, the experimental Hall number ${ }^{3}$ is in excess of the paramagnetic band theory value $e^{6}$ for temperatures above 150 $\mathrm{K}$, again indicating the presence of an inelastic scattering contribution (which could be of magnetic origin as well). Calculation of the $T$ dependence of the Hall number, though, is technically challenging 12 since it involves going beyond the Boltzmann approximation, so we do not consider this further here.

On the other hand, our results do indicate a large pseudogap in the strong coupling limit. We note that there are examples of quantum critical points where Fermi liquid theory is known to break down, and where nesting may be playing an important role, such as in the case of the bilayer ruthenate $\mathrm{Sr}_{3} \mathrm{Ru}_{2} \mathrm{O}_{7}{ }^{20}$. Moreover, magnetic incommensurability is seen in most quantum critical heavy fermion systems, such as Au doped $\mathrm{CeCu}_{6}{ }^{21}$. It is possible that the results presented here, which were derived for the case where a strong Fermi surface rearrangment takes place at the quantum critical point, are quite relevant for these systems. Based on this, we suggest that pseudogap effects be searched for in heavy fermion quantum critical systems.

We would like to thank the authors of Ref. [3, particularly Tom Rosenbaum, for communicating their work to us prior to publication. This work was supported by the U. S. Dept. of Energy, Office of Science, under Contract No. W-31-109-ENG-38. CP would like to thank the hospitality of ANL, and MN that of the SPhT, while this work was in progress.
${ }^{1}$ G. R. Stewart, Rev. Mod. Phys. 73, 797 (2001).

2 P. Coleman, C. Pépin, Q. Si, and R. Ramazashvili, J. Phys. Cond. Matter 13, R723 (2001).

3 A. Yeh et al., Nature 419, 459 (2002).

4 E. Fawcett, Rev. Mod. Phys. 60, 209 (1988); E. Fawcett, H. L. Alberts, V. Yu. Galkin, D. R. Noakes, J. V. Yakhmi, ibid 66, 25 (1994).

5 S. M. Hayden et al., Phys. Rev. Lett. 84, 999 (2000); O. Stockert et al., unpublished.

${ }^{6}$ M. R. Norman, Q. Si, Ya. B. Bazaliy, and R. Ramazashvili, Phys. Rev. Lett. 90, 116601 (2003).

7 P. A. Lee, T. M. Rice, and P. W. Anderson, Phys. Rev. Lett. 31, 462 (1973).

8 A. Shibatani, K. Motizuki, and T. Nagamiya, Phys. Rev. 177, 984 (1969); K. Machida and M. Fujita, Phys. Rev. B 30, 5284 (1984).

9 H. Hasegawa, J. Low Temp. Phys. 31, 475 (1978).

10 T. M. Rice, Phys. Rev. B 2, 3619 (1970).

11 M. Lee, A. Husmann, T.F. Rosenbaum, and G. Aeppli, to be published.

12 T. Beaulac, P. B. Allen, and F. J. Pinski, Phys. Rev. B 26, 1549 (1982).

13 H. Sato and K. Maki, Intl. J. Magn. 4, 163 (1973).

14 G. G. Lonzarich and L. Taillefer, J. Phys. C 18, 4339 (1985).

15 T. Moriya, Spin Fluctuations in Itinerant Electron Magnetism (Springer-Verlag, Berlin, 1985), p. 66.

16 In the presence of disorder, $T_{N}$ can be driven to zero in the flat case since nesting is smeared out. This is unlikely to be the case for chromium, in that $T_{N}$ can be driven to zero by pressure instead of doping ${ }^{4.11}$.

17 N. F. Berk and J. R. Schrieffer, Phys. Rev. Lett. 17, 433 (1966); D. R. Penn, Phys. Lett. 25A, 269 (1967).

18 Y. M. Vilk, Phys. Rev. B 55, 3870 (1997); Y. M. Vilk and A.-M. S. Tremblay, J. Phys. I France 7, 1309 (1997).

19 J. Schafer et al., Phys. Rev. Lett. 83, 2069 (1999).

20 R. S. Perry et al., Phys. Rev. Lett. 86, 2661 (2001); L. Capogna et al., Phys. Rev. B 67, 012504 (2003). 
21 A. Schröder et al., Nature 407, 351 (2000). 